\title{
PT-Symmetric Matrix Quasi-Exactly Solvable Razhavi Potential
}

\author{
Ancilla Nininahazwe \\ Institut de Pédagogie Appliquée, Université du Burundi, Bujumbura, Burundi \\ Email: nininaha@yahoo.fr
}

How to cite this paper: Nininahazwe, A. (2020) PT-Symmetric Matrix Quasi-Exactly Solvable Razhavi Potential. Open Journal of Microphysics, 10, 9-20.

https://doi.org/10.4236/ojm.2020.102002

Received: February 14, 2020

Accepted: May 22, 2020

Published: May 25, 2020

Copyright ( 2020 by author(s) and Scientific Research Publishing Inc. This work is licensed under the Creative Commons Attribution International License (CC BY 4.0).

http://creativecommons.org/licenses/by/4.0/

\begin{abstract}
A $P T$-symmetric Hamiltonian associated with a trigonometric Razhavi potential is analyzed. Along the same lines of the general quasi-exactly solvable analytic method considered in the [1] [2] [3], three necessary and sufficient algebraic conditions for this Hamiltonian to have a finite-dimensional invariant vector space are established. This $P T$-symmetric $2 \times 2$-matrix Hamiltonian is called quasi-exactly solvable (QES).
\end{abstract}

\section{Keywords}

PT-Symmetric Hamiltonian, Trigonometric Potential, QES analytic Method, Invariant Vector Space

\section{Introduction}

In quantum physics, one of the main mathematical problems consists in constructing the set of eigenvalues of a linear operator defined on a suitable domain of a Hilbert space. In most cases, this type of problem cannot be explicitly solved, that is to say that the spectrum of the Hamiltonian cannot be found algebraically. However, in few cases, some of which turn out to be physically fundamental, the spectrum can indeed be computed explicitly. These cases are so called completely solvable (or exactly solvable). The example of this kind is the harmonic quantum oscillator.

In the last few years, the intermediate class between exactly solvable operators and non solvable operators has been discovered. This class is called quasi-exactly solvable. It means that a finite part of eigenvalues associated to this type of operators can be found algebraically [1]-[10].

Another concept that we consider throughout this paper is the $P T$-symmetric operator. It means that this operator is invariant under the combination of the 
parity operator $P$ and the time-reversal operator $T$. Note that the Hamiltonian we analyze is both QES and PT-symmetric.

This paper is organized as follows:

In Section 2, based on the Ref. [1] [2] [3], we briefly recall the general QES analytic method used to investigate the quasi-exact solvability of $2 \times 2$-matrix Hamiltonians.

In Section 3, we show in details that the non hermitian Razhavi Hamiltonian is invariant under the combined $P T$-symmetry.

In Section 4, along the same lines as in the Refs. [1] [2] [3], we apply the QES analytic method in order to prove the quasi-exact solvability of the $2 \times 2$-matrix Hamiltonian depending on the $P T$-symmetric potential. Three necessary and sufficient QES conditions for the $2 \times 2$-matrix trigonometric Hamiltonian to have a finite dimensional invariant vector space will be established for two cases: $\psi=\left(p_{n}, \cos x q_{n-1}\right)^{t}$ and $\psi=\left(p_{n-1} \cos x, q_{n}\right)^{t}$.

\section{QES Analytic Method}

Taking account to the same lines as in the Refs. [1] [2] [3], we recall a general method to check whether a $2 \times 2$-matrix differential operator $H$ (in a variable $x$ ) preserves a vector space whose components are polynomials.

Consider the $2 \times 2$-matrix Hamiltonian of the following form [1] [2] [3]:

$$
H=\left(\begin{array}{cc}
-\frac{\mathrm{d}^{2}}{\mathrm{~d} x^{2}}+V_{11}(x) & x^{\delta} \\
x^{\delta^{\prime}} & -\frac{\mathrm{d}^{2}}{\mathrm{~d} x^{2}}+V_{22}(x)
\end{array}\right)
$$

where

$$
V(x)=\left(\begin{array}{cc}
V_{11}(x) & x^{\delta} \\
x^{\delta^{\prime}} & V_{22}(x)
\end{array}\right), \quad \delta=0,1,2, \quad \delta^{\prime}=1-\delta, \quad V_{12}(x)=x^{\delta}, \quad V_{21}(x)=x^{\delta^{\prime}}
$$

$V(x)$ is the potential associated to the Hamiltonian $H$ given by this above relation (1).

A gauge transformation and a change of variable on the Hamiltonian $H$ lead to the following Hamiltonian called the gauge one

$$
\tilde{H}=\phi^{-1} H \phi
$$

which can be written in his components as follows

$$
\tilde{H}=\tilde{H}_{1}+\tilde{H}_{0}+\tilde{H}_{-1} .
$$

More precisely, the diagonal components of $\tilde{H}_{1}$ are differential operators and the off-diagonal components $\left(\tilde{H}_{1}\right)_{12}$ and $\left(\tilde{H}_{1}\right)_{21}$ are respectively proportional to $x^{\delta}$ and $x^{\delta^{\prime}}$. The operators $\tilde{H}_{0}$ and $\tilde{H}_{-1}$ have lower degrees in all their components than the corresponding components in $\tilde{H}_{1}$.

Note that the invariant vector space of the Hamiltonian $\tilde{H}$ has the following form [1] [2] [3]: 


$$
W=\left\{\left(\begin{array}{c}
p_{n} \\
q_{n}
\end{array}\right)\right\}, \quad m=n-\delta+1 \text { and } n, m \in \mathrm{N}
$$

In order to obtain the QES conditions for $\tilde{H}$, the generic vector of the above vector space is of the form

$$
\varphi=\left(\begin{array}{c}
\alpha_{0} x^{n}+\alpha_{1} x^{n-1} \\
\beta_{0} x^{n-\delta+1}+\beta_{1} x^{n-\delta}
\end{array}\right),
$$

where $\alpha_{i}, \beta_{i}(i=0,1)$ are complex parameters. As a consequence the $2 \times 2$ -matrices $M_{1}, \tilde{M}_{1}, M_{0}$ are defined by

$$
\begin{gathered}
\tilde{H}_{1}\left(\begin{array}{c}
\alpha_{0} x^{n} \\
\beta_{0} x^{n-\delta+1}
\end{array}\right)=\operatorname{diag}\left(x^{n+1}, x^{n-\delta+2}\right) M_{1}\left(\begin{array}{c}
\alpha_{0} \\
\beta_{0}
\end{array}\right), \\
\tilde{H}_{1}\left(\begin{array}{c}
\alpha_{1} x^{n-1} \\
\beta_{1} x^{n-\delta}
\end{array}\right)=\operatorname{diag}\left(x^{n}, x^{n-\delta+1}\right) \tilde{M}_{1}\left(\begin{array}{c}
\alpha_{1} \\
\beta_{1}
\end{array}\right), \\
\tilde{H}_{0}\left(\begin{array}{c}
\alpha_{0} x^{n} \\
\beta_{0} x^{n-\delta+1}
\end{array}\right)=\operatorname{diag}\left(x^{n}, x^{n-\delta+1}\right) M_{0}\left(\begin{array}{c}
\alpha_{0} \\
\beta_{0}
\end{array}\right) .
\end{gathered}
$$

The three necessary and sufficient QES conditions for $\tilde{H}$ to have an invariant vector space are

$$
\begin{aligned}
\text { 1) } M_{1}\left(\begin{array}{c}
\alpha_{0} \\
\beta_{0}
\end{array}\right)=\left(\begin{array}{l}
0 \\
0
\end{array}\right), \\
\text { 2) } \tilde{M}_{1}^{t}\left(\begin{array}{c}
-\beta_{0} \\
\alpha_{0}
\end{array}\right)=\left(\begin{array}{l}
0 \\
0
\end{array}\right) \text {, } \\
\text { 3) } M_{0}\left(\begin{array}{c}
1 \\
\frac{\beta_{0}}{\alpha_{0}}
\end{array}\right)=\Lambda\left(\begin{array}{l}
1 \\
\frac{\beta_{0}}{\alpha_{0}}
\end{array}\right) \text {. }
\end{aligned}
$$

In the next step, we will apply in a systematic lines of this QES analytic method in order to construct a $2 \times 2$-matrix QES Hamiltonian associated to a PT-symmetric and trigonometric potential.

\section{PT-Symmetric and Trigonometric QES Potential}

In this section, we consider the PT-symmetric and trigonometric $2 \times 2$-matrix Hamiltonian [1] [2]:

$$
H=\left(\begin{array}{cc}
-\frac{\mathrm{d}^{2}}{\mathrm{~d} x^{2}}+(\rho \cos 2 x-i M)^{2}+A & H_{12} \\
H_{21} & -\frac{\mathrm{d}^{2}}{\mathrm{~d} x^{2}}+(\rho \cos 2 x-i M)^{2}+\tilde{A}
\end{array}\right),
$$

where $A, \tilde{A}, M, \tilde{M}, \rho$ are free real parameters.

The non diagonal components of the Hamiltonian $H$ (i.e. $H_{12}, H_{21}$ ) are written as follows

$$
\begin{aligned}
& H_{12}=C \cos x, \\
& H_{12}=\tilde{C} \cos x,
\end{aligned}
$$


with $C$ and $\tilde{C}$ are the coupling constants.

Now, we can reveal the PT-symmetry property of the trigonometric non hermitian $2 \times 2$-matrix potential associated to the above Hamiltonian given by the Equation (8)

$$
\begin{gathered}
V=\left(\begin{array}{ll}
V_{11} & V_{12} \\
V_{21} & V_{22}
\end{array}\right), \\
V=\left(\begin{array}{cc}
(\rho \cos 2 x-i M)^{2}+A & C \cos x \\
\tilde{C} \cos x & (\rho \cos 2 x-i M)^{2}+\tilde{A}
\end{array}\right)
\end{gathered}
$$

In order to prove that this above potential $V$ is invariant under the combined action of the parity operator $P$ and the time-reversal operator $T$ (i.e. the combined $P T$-symmetry), the potential $V$ has to satisfy the following relation [1] [2] [4]:

$$
V^{P T}=V
$$

Note that the time-reversal operator $T$ reverses the sign of the complex number $i$ :

$$
T i T^{-1}=-i
$$

while the parity operator replaces $x$ by $\frac{\pi}{2}-x$ leading to

$$
V^{P T}=\left(\begin{array}{cc}
{[\rho \cos (\pi-2 x)+i M]^{2}+A} & C \cos \left(\frac{\pi}{2}-x\right) \\
\tilde{C} \cos \left(\frac{\pi}{2}-x\right) & {[\rho \cos (\pi-2 x)+i \tilde{M}]^{2}+\tilde{A}}
\end{array}\right)
$$

with

$$
\begin{gathered}
V_{11}^{P T}(x)=[\rho \cos (\pi-2 x)+i M]^{2}+A, \\
V_{11}^{P T}(x)=\rho^{2}(\cos \pi \cos 2 x+\sin \pi \sin 2 x)^{2} \\
+2 i \rho M(\cos \pi \cos 2 x+\sin \pi \sin 2 x)-M^{2}+A, \\
V_{11}^{P T}(x)=\rho^{2} \cos ^{2} 2 x-2 i \rho M \cos 2 x-M^{2}+A, \\
V_{11}^{P T}(x)=(\rho \cos 2 x-i M)^{2}+A, \\
V_{11}^{P T}(x)=V_{11}(x) .
\end{gathered}
$$

After the similar algebraic manipulations used in the previous equation, one can easily check that the other three components elements of the potential $V^{P T}$ given by the Equation (12) satisfy the following relations:

$$
\begin{aligned}
& V_{12}^{P T}(x)=V_{12}(x), \\
& V_{21}^{P T}(x)=V_{21}(x), \\
& V_{22}^{P T}(x)=V_{22}(x) .
\end{aligned}
$$

Referring to the previous relations (13) and (14), we are allowed to write that 


$$
V^{P T}(x)=V(x) .
$$

Thus, taking account to this Equation (15), we can conclude that the potential $V(x)$ is invariant under the combined action of the parity operator $P$ and the time-reversal operator $T$. In other words, the potential $V(x)$ given by the Equation (10) is PT-symmetric. As a consequence, it is this $P T$-invariant symmetry property which explains the reality of some of the eigenvalues associated to the above trigonometric potential $V(x)$ [1] [2] [3].

\section{PT-Symmetric Hamiltonian and His Quasi-Exact Solvability}

In this section, we apply the QES analytic method established in the section 2 to prove that the PT-symmetric Hamiltonian given by the Equation (8) is quasi-exactly solvable.

In order to reveal the quasi-exact solvability of the previous Hamiltonian $H$, it is therefore necessary to transform $H$ with the gauge function as follows [1] [2] [3]:

$$
\begin{gathered}
\tilde{H}=R^{-1} H R, \\
\tilde{H}=\mathrm{e}^{-\theta \cos 2 x}\left(\begin{array}{cc}
z^{-\varepsilon}(1-z)^{-\phi} & 0 \\
0 & z^{-\tilde{\varepsilon}}(1-z)^{-\tilde{\phi}}
\end{array}\right) H \mathrm{e}^{\theta \cos 2 x}\left(\begin{array}{cc}
z^{\varepsilon}(1-z)^{\phi} & 0 \\
0 & z^{\tilde{\varepsilon}}(1-z)^{\tilde{\phi}}
\end{array}\right),
\end{gathered}
$$

where the gauge function is written as follows

$$
R(x)=\mathrm{e}^{\theta \cos 2 x}\left(\begin{array}{cc}
z^{\varepsilon}(1-z)^{\phi} & 0 \\
0 & z^{\tilde{\varepsilon}}(1-z)^{\tilde{\phi}}
\end{array}\right)
$$

and the gauge Hamiltonian is of the following form

$$
\tilde{H}=\left(\begin{array}{ll}
\tilde{H}_{11} & \tilde{H}_{12} \\
\tilde{H}_{21} & \tilde{H}_{22}
\end{array}\right) .
$$

The next step is to perform the change in the variable $Z$

$$
z=\frac{\cos (2 x+1)}{2}
$$

in order to find the final form of the above gauge Hamiltonian.

In the following, we will consider in details the cases $H_{12}=C \cos x$ and $H_{12}=\tilde{C} \cos x$ which are the non diagonal elements of the matrix PT-symmetric Hamiltonian $H$ given by the Equation (8).

In this case, the possible values for the parameters $\varepsilon, \tilde{\varepsilon}, \phi, \tilde{\phi}$ allow two cases for the wave function, $\psi=\left(\psi_{1}, \psi_{2}\right)^{t}$ which have the following forms:

Case 1:

$$
\psi=\left(p_{n}, \cos x q_{n-1}\right)^{t},
$$

Case 2:

$$
\psi=\left(p_{n-1} \cos x, q_{n}\right)^{t} .
$$


If the parameter $\theta$ of the gauge function of the Equation (17) is chosen as $\theta=i \frac{\rho}{2}$, the components of the gauge Hamiltonian $\tilde{H}(z)$ are written as follows:

$$
\begin{aligned}
& \tilde{H}_{11}=-4 z(1-z) \frac{\mathrm{d}^{2}}{\mathrm{~d} z^{2}}+2[2 z-1-4(1-z) \varepsilon+4 \phi z] \frac{\mathrm{d}}{\mathrm{d} z}+\rho^{2}-M^{2} \\
&+8 \phi \varepsilon+2 \varepsilon+2 \phi+A-8 i \rho\left[z(1-z) \frac{\mathrm{d}}{\mathrm{d} z}+\varepsilon(1-z)-\phi z+\frac{M-1}{4}(2 z-1)\right] \\
& \tilde{H}_{12}=z^{\tilde{\varepsilon}-\varepsilon}(1-z)^{\tilde{\phi}-\phi} C \cos x \tilde{H}_{21}=z^{\varepsilon-\tilde{\varepsilon}}(1-z)^{\phi-\tilde{\phi}} \tilde{C} \cos x \\
& \tilde{H}_{22}=-4 z(1-z) \frac{\mathrm{d}^{2}}{\mathrm{~d} z^{2}}+2[2 z-1-4(1-z) \tilde{\varepsilon}+4 \tilde{\phi} z] \frac{\mathrm{d}}{\mathrm{d} z}+\rho^{2}-\tilde{M}^{2} \\
&+8 \tilde{\phi} \tilde{\varepsilon}+2 \tilde{\varepsilon}+2 \tilde{\phi}+\tilde{A}-8 i \rho\left[z(1-z) \frac{\mathrm{d}}{\mathrm{d} z}+\tilde{\varepsilon}(1-z)-\tilde{\phi} z+\frac{\tilde{M}-1}{4}(2 z-1)\right]
\end{aligned}
$$

Taking account to the change in variable $z$ given by the Equation (19), one can easily find that

$$
\cos x=z^{\frac{1}{2}}
$$

\subsection{Case 1: $\psi=\left(p_{n}, \cos x q_{n-1}\right)^{t}$}

The function $\psi$ can be written as follows:

$$
\psi=\left(\begin{array}{cc}
1 & 0 \\
0 & \cos x
\end{array}\right)\left(\begin{array}{c}
p_{n} \\
q_{n-1}
\end{array}\right)
$$

Referring to the Equation (23), one can find that

$$
\left(\begin{array}{cc}
z^{\varepsilon}(1-z)^{\phi} & 0 \\
0 & z^{\tilde{\varepsilon}}(1-z)^{\tilde{\phi}}
\end{array}\right)=\left(\begin{array}{cc}
1 & 0 \\
0 & \cos x
\end{array}\right)
$$

only if

$$
\varepsilon=\phi=\tilde{\phi}=0, \tilde{\varepsilon}=\frac{1}{2} .
$$

Considering the relation (23) and replacing the parameters $\varepsilon, \phi, \tilde{\phi}, \tilde{\varepsilon}$ respectively by their values as $\varepsilon=\phi=\tilde{\phi}=0, \tilde{\varepsilon}=\frac{1}{2}$ and after some algebraic manipulations, the components of the gauge Hamiltonian given by the Equation (22) can be written in the following final form:

$$
\begin{gathered}
\tilde{H}_{11}=-4 z \frac{\mathrm{d}^{2}}{\mathrm{~d} z^{2}}+4 z^{2} \frac{\mathrm{d}^{2}}{\mathrm{~d} z^{2}}+4 z \frac{\mathrm{d}}{\mathrm{d} z}-2 \frac{\mathrm{d}}{\mathrm{d} z}+\rho^{2}-M^{2}+A \\
-8 i \rho z \frac{\mathrm{d}}{\mathrm{d} z}+8 i \rho z^{2} \frac{\mathrm{d}}{\mathrm{d} z}-4 i \rho(M-1) z+2 i \rho(M-1) \\
\tilde{H}_{12}=C z \\
\tilde{H}_{21}=\tilde{C},
\end{gathered}
$$




$$
\begin{aligned}
\tilde{H}_{22}= & -4 z \frac{\mathrm{d}^{2}}{\mathrm{~d} z^{2}}+4 z^{2} \frac{\mathrm{d}^{2}}{\mathrm{~d} z^{2}}+8 z \frac{\mathrm{d}}{\mathrm{d} z}-6 \frac{\mathrm{d}}{\mathrm{d} z}+\rho^{2}-\tilde{M}^{2}+1+\tilde{A} \\
& -8 i \rho z \frac{\mathrm{d}}{\mathrm{d} z}+8 i \rho z^{2} \frac{\mathrm{d}}{\mathrm{d} z}-4 i \rho(\tilde{M}-2) z+2 i \rho(\tilde{M}-3)
\end{aligned}
$$

Referring to the Equation (3) of the QES analytic method, the above Hamiltonian given by the Equation (26) can be written as follows

$$
\tilde{H}=\tilde{H}_{1}+\tilde{H}_{0}+\tilde{H}_{-1}
$$

where

$$
\tilde{H}_{1}=\left(\begin{array}{cc}
8 i \rho\left[z^{2} \frac{\mathrm{d}}{\mathrm{d} z}-\left(\frac{M-1}{2}\right) z\right] & 0 \\
\tilde{C} & 8 i \rho\left[z^{2} \frac{\mathrm{d}}{\mathrm{d} z}-\left(\frac{\tilde{M}-2}{2}\right) z\right]
\end{array}\right)
$$

$$
\begin{gathered}
\tilde{H}_{0}=\left(\begin{array}{cc}
4 z^{2} \frac{\mathrm{d}^{2}}{\mathrm{~d} z^{2}}+(4-8 i \rho) z \frac{\mathrm{d}}{\mathrm{d} z}+\rho^{2}-M^{2}+A+2 i \rho(M-1) & C z \\
O & 4 z^{2} \frac{\mathrm{d}^{2}}{\mathrm{~d} z^{2}}+(8-8 i \rho) z \frac{\mathrm{d}}{\mathrm{d} z}+\rho^{2}-\tilde{M}^{2}+\tilde{A}+1+2 i \rho(M-3)
\end{array}\right) \\
\tilde{H}_{-1}=\left(\begin{array}{cc}
-4 z \frac{\mathrm{d}^{2}}{\mathrm{~d} z^{2}}-2 \frac{\mathrm{d}}{\mathrm{d} z} & 0 \\
0 & -4 z \frac{\mathrm{d}^{2}}{\mathrm{~d} z^{2}}-6 \frac{\mathrm{d}}{\mathrm{d} z}
\end{array}\right)
\end{gathered}
$$

Note that the action of these above three gauge components of $\tilde{H}$ given by the Equation (27) on the wave function $\psi$ given by the relation (20) leads to the following expressions:

$$
\begin{aligned}
& \tilde{H}_{1}\left(\begin{array}{c}
z^{n} \\
z^{n-1}
\end{array}\right) \cong\left(\begin{array}{c}
z^{n+1} \\
z^{n}
\end{array}\right), \\
& \tilde{H}_{0}\left(\begin{array}{c}
z^{n} \\
z^{n-1}
\end{array}\right) \cong\left(\begin{array}{c}
z^{n} \\
z^{n-1}
\end{array}\right), \\
& \tilde{H}_{-1}\left(\begin{array}{c}
z^{n} \\
z^{n-1}
\end{array}\right) \cong\left(\begin{array}{c}
z^{n-1} \\
z^{n-2}
\end{array}\right) .
\end{aligned}
$$

After some algebraic manipulations, one can easily obtain the $2 \times 2$-matrices $M_{1}, \tilde{M}_{1}, M_{0}$ respectively as follows [1] [2] [4]:

$$
\begin{aligned}
& \tilde{H}_{1}\left(\begin{array}{c}
\alpha_{0} z^{n} \\
\beta_{0} z^{n-1}
\end{array}\right)=\operatorname{diag}\left(z^{n+1}, z^{n}\right) M_{1}\left(\begin{array}{c}
\alpha_{0} \\
\beta_{0}
\end{array}\right), \\
& \tilde{H}_{1}\left(\begin{array}{c}
\alpha_{1} z^{n-1} \\
\beta_{1} z^{n-2}
\end{array}\right)=\operatorname{diag}\left(z^{n}, z^{n-1}\right) \tilde{M}_{1}\left(\begin{array}{c}
\alpha_{1} \\
\beta_{1}
\end{array}\right), \\
& \tilde{H}_{0}\left(\begin{array}{c}
\alpha_{0} z^{n} \\
\beta_{0} z^{n-1}
\end{array}\right)=\operatorname{diag}\left(z^{n}, z^{n-1}\right) M_{0}\left(\begin{array}{c}
\alpha_{0} \\
\beta_{0}
\end{array}\right) .
\end{aligned}
$$

Replacing in the relations (29) respectively $\tilde{H}_{1}$ and $\tilde{H}_{0}$ by their expressions given by the Equation (27), one can easily deduce the final form of the three ma- 
trices $M_{1}, \tilde{M}_{1}, M_{0}$ as follows

$$
\begin{aligned}
& M_{1}=\left(\begin{array}{cc}
8 i \rho\left[n-\left(\frac{M-1}{2}\right)\right] & 0 \\
\tilde{C} & 8 i \rho\left(n-\frac{\tilde{M}}{2}\right)
\end{array}\right), \\
& \tilde{M}_{1}=\left(\begin{array}{cc}
8 i \rho\left[n-\left(\frac{M+1}{2}\right)\right] & 0 \\
\tilde{C} & 8 i \rho\left[n-\left(\frac{\tilde{M}}{2}+1\right)\right]
\end{array}\right), \\
& M_{0}=\left(\begin{array}{cc}
n^{2}+3 n-8 i \rho n+\rho^{2}-M^{2}+A+2 i \rho(M-1) & -C \\
0 & n^{2}+5 n-5-8 i \rho n+\rho^{2}-\tilde{M}^{2}+\tilde{A}+2 i \rho(\tilde{M}+1)
\end{array}\right) \text {. }
\end{aligned}
$$

Our purpose is to establish the three necessary and sufficient QES conditions for the gauge Hamiltonian given by the Equation (26) to have a finite dimensional invariant vector space [1] [2] [3] [4]:

1) The first QES condition is obtained as follows

$$
\begin{gathered}
M_{1}\left(\begin{array}{l}
\alpha_{0} \\
\beta_{0}
\end{array}\right)=\left(\begin{array}{l}
0 \\
0
\end{array}\right), \\
\operatorname{det} M_{1}=0, \\
\operatorname{det}\left(\begin{array}{c}
8 i \rho\left[n-\left(\frac{M-1}{2}\right)\right] \\
\tilde{C}\left(n-\frac{\tilde{M}}{2}\right)
\end{array}\right)=0, \\
\tilde{M}=\frac{-4 n^{2}+2 n M-2 n}{-2 n+M-1}
\end{gathered}
$$

2) The second QES condition is of the following the form

$$
\begin{gathered}
\operatorname{det} \tilde{M}_{1}=0, \\
M=\frac{-4 n^{2}+6 n+2 n \tilde{M}-\tilde{M}-2}{-2 n+\tilde{M}+2}
\end{gathered}
$$

3) The third and final QES condition is found by the condition involving the matrix $M_{0}$ as follows

$$
M_{0}\left(\begin{array}{c}
1 \\
\frac{\beta_{0}}{\alpha_{0}}
\end{array}\right)=\Lambda\left(\begin{array}{c}
1 \\
\frac{\beta_{0}}{\alpha_{0}}
\end{array}\right),
$$

where $\Lambda$ is a constant

Taking account to the first QES condition, the expression $\frac{\beta_{0}}{\alpha_{0}}$ is obtained as follows

$$
M_{1}\left(\begin{array}{l}
\alpha_{0} \\
\beta_{0}
\end{array}\right)=\left(\begin{array}{l}
0 \\
0
\end{array}\right)
$$




$$
\frac{\beta_{0}}{\alpha_{0}}=\frac{-\tilde{C}}{8 i \rho\left(n-\frac{\tilde{M}}{2}\right)}
$$

Replacing the expression (30) and (34) in the relation (33) and after some algebraic manipulations, one can easily find the third QES condition as

$$
\tilde{A}=-2 n+5+\tilde{M}^{2}-M^{2}+A-2 i \rho(\tilde{M}-M+2)-\frac{C \tilde{C}}{8 i \rho\left(n-\frac{\tilde{M}}{2}\right)}
$$

Taking account to three necessary and sufficient QES conditions given by the relations (31), (32) and (35), one can conclude that the gauge Hamiltonian $\tilde{H}$ given by the equation (26) (therefore the extended Razhavi Hamiltonian $H$ given by the equation (8)) is quasi-exactly solvable. In other words, a finite part of the eigenvalues associated to the gauge Hamiltonian can be found algebraically.

\subsection{Case 2: $\psi=\left(p_{n-1} \cos x, q_{n}\right)^{t}$}

Along the same lines used in the previous case (i.e. case 1), this case 2 can be studied.

Taking account to the gauge Hamiltonian $\tilde{H}$ given by the Equation (22) and the change variable (23), the four components of $\tilde{H}$ are written as follows

$$
\begin{array}{r}
\tilde{H}_{11}=-4 z \frac{\mathrm{d}^{2}}{\mathrm{~d} z^{2}}+4 z^{2} \frac{\mathrm{d}^{2}}{\mathrm{~d} z^{2}}+8 z \frac{\mathrm{d}}{\mathrm{d} z}-6 \frac{\mathrm{d}}{\mathrm{d} z}+\rho^{2}-M^{2}+1+A \\
-8 i \rho z \frac{\mathrm{d}}{\mathrm{d} z}+8 i \rho z^{2} \frac{\mathrm{d}}{\mathrm{d} z}-4 i \rho(M-2) z+2 i \rho(M-3), \\
\tilde{H}_{12}=C \\
\tilde{H}_{21}=\tilde{C} z \\
\tilde{H}_{22}=-4 z \frac{\mathrm{d}^{2}}{\mathrm{~d} z^{2}}+4 z^{2} \frac{\mathrm{d}^{2}}{\mathrm{~d} z^{2}}+4 z \frac{\mathrm{d}}{\mathrm{d} z}-2 \frac{\mathrm{d}}{\mathrm{d} z}+\rho^{2}-\tilde{M}^{2}+\tilde{A} \\
-8 i \rho z \frac{\mathrm{d}}{\mathrm{d} z}+8 i \rho z^{2} \frac{\mathrm{d}}{\mathrm{d} z}-4 i \rho(\tilde{M}-1) z+2 i \rho(\tilde{M}-1)
\end{array}
$$

where $\phi=\tilde{\varepsilon}=\tilde{\phi}=0$ and $\varepsilon=\frac{1}{2}$.

Note that the other form of the gauge Hamiltonian given by the above equation (36) is as follows

$$
\tilde{H}=\tilde{H}_{1}+\tilde{H}_{0}+\tilde{H}_{-1}
$$

Applying these three components of the gauge Hamiltonian $\tilde{H}$ as

$$
\begin{aligned}
& \tilde{H}_{1}\left(\begin{array}{c}
z^{n-1} \\
z^{n}
\end{array}\right) \cong\left(\begin{array}{c}
z^{n} \\
z^{n+1}
\end{array}\right), \\
& \tilde{H}_{0}\left(\begin{array}{c}
z^{n-1} \\
z^{n}
\end{array}\right) \cong\left(\begin{array}{c}
z^{n-1} \\
z^{n}
\end{array}\right),
\end{aligned}
$$




$$
\begin{aligned}
& \tilde{H}_{-1}\left(\begin{array}{c}
z^{n-1} \\
z^{n}
\end{array}\right) \cong\left(\begin{array}{c}
z^{n-2} \\
z^{n-1}
\end{array}\right), \\
& \tilde{H}_{1}=\left(\begin{array}{cc}
8 i \rho z^{2} \frac{\mathrm{d}}{\mathrm{d} z}-4 i \rho(M-2) z & C \\
0 & 8 i \rho z^{2} \frac{\mathrm{d}}{\mathrm{d} z}-4 i \rho(\tilde{M}-1) z
\end{array}\right), \\
& \tilde{H}_{-1}=\left(\begin{array}{cc}
-4 z \frac{\mathrm{d}^{2}}{\mathrm{~d} z}-6 \frac{\mathrm{d}}{\mathrm{d} z} & 0 \\
0 & -4 z \frac{\mathrm{d}^{2}}{\mathrm{~d} z}-2 \frac{\mathrm{d}}{\mathrm{d} z}
\end{array}\right)
\end{aligned}
$$

After some algebraic manipulations, the $2 \times 2$-matrices $M_{1}, \tilde{M}_{1}, M_{0}$ can be computed as follows [1] [2] [4]:

$$
\begin{aligned}
\tilde{H}_{1}\left(\begin{array}{c}
\alpha_{0} z^{n-1} \\
\beta_{0} z^{n}
\end{array}\right) & =\operatorname{diag}\left(z^{n}, z^{n+1}\right) M_{1}\left(\begin{array}{c}
\alpha_{0} \\
\beta_{0}
\end{array}\right), \\
\tilde{H}_{1}\left(\begin{array}{c}
\alpha_{1} z^{n-2} \\
\beta_{1} z^{n-1}
\end{array}\right) & =\operatorname{diag}\left(z^{n-1}, z^{n}\right) \tilde{M}_{1}\left(\begin{array}{c}
\alpha_{1} \\
\beta_{1}
\end{array}\right), \\
\tilde{H}_{0}\left(\begin{array}{c}
\alpha_{0} z^{n-1} \\
\beta_{0} z^{n}
\end{array}\right) & =\operatorname{diag}\left(z^{n-1}, z^{n}\right) M_{0}\left(\begin{array}{c}
\alpha_{0} \\
\beta_{0}
\end{array}\right) .
\end{aligned}
$$

And these above three relations (39) lead respectively to these following three matrices:

$$
\begin{gathered}
M_{1}=\left(\begin{array}{cc}
4 i \rho(2 n-M) & C \\
0 & 8 i \rho n-4 i \rho(\tilde{M}-1)
\end{array}\right), \\
\tilde{M}_{1}=\left(\begin{array}{cc}
8 i \rho(n-1)-4 i \rho M & C \\
0 & 4 i \rho(n-1)-4 i \rho \tilde{M}
\end{array}\right), \\
M_{0}=\left(\begin{array}{cc}
4 n^{2}-4 n+1+\rho^{2}-M^{2}+A-8 i \rho n+2 i \rho M+2 i \rho & 0 \\
\tilde{C} & 4 n^{2}+\rho^{2}-\tilde{M}^{2}+\tilde{A}-8 i \rho n+2 i \rho \tilde{M}-2 i \rho
\end{array}\right)
\end{gathered}
$$

Referring to the same lines of the case 1 (i.e. studied in the previous step), one can easily find the following three necessary and sufficient QES conditions for the gauge Hamiltonian given by the Equation (36) to have a finite dimensional invariant vector space [1] [2] [3]:

1) The first QES condition is

$$
\tilde{M}=\frac{4 n^{2}+2 n(1-M)-M}{2 n-M}
$$

2) The second QES condition is as follows 


$$
M=\frac{-4 n^{2}+6 n+2 \tilde{M}(n-1)-2}{-n+\tilde{M}+1}
$$

3) The third and the final QES condition is

$$
\tilde{C}=\frac{[-4 i \rho(2 n-M)]\left[-4 n^{2}-\rho^{2}+\tilde{M}^{2}-\tilde{A}+8 i \rho n-2 i \rho(\tilde{M}-1)+1\right]}{C}
$$

Taking account to the three necessary and sufficient QES conditions given by It means that the gauge Hamiltonian $\tilde{H}$ has a finite dimensional vector space which is invariant under its action. The relations (40), (41) and (42), one can conclude that the gauge Hamiltonian $\tilde{H}$ (therefore $\mathrm{H}$ ) given by the Equation (36) is quasi-exactly solvable.

\section{Conclusion}

In this paper, we have applied the general QES analytic method established in the Ref. [1] in order to prove that the $P T$-symmetric $2 \times 2$-matrix Razhavi Hamiltonian is quasi-exactly solvable. It means that a finite part of eigenvalues associated to this Razhavi Hamiltonian can be computed algebraically. We have considered two cases for the wave function: $\psi=\left(p_{n}, \cos x q_{n-1}\right)^{t}$ and $\psi=\left(p_{n-1} \cos x, q_{n}\right)^{t}$. The three necessary and sufficient QES conditions for the $P T$-symmetric $2 \times 2$-matrix trigonometric Hamiltonian to have a finite vector space invariant under its action are found algebraically.

\section{Acknowledgements}

I thank Pr. Yves Brihaye of useful discussions.

\section{Conflicts of Interest}

The author declares no conflicts of interest regarding the publication of this paper.

\section{References}

[1] Brihaye, Y., Nininahazwe, A. and Mandal, B.P. (2007) PT-Symmetric, Quasi-Exactly Solvable Matrix Hamiltonians. Journal of Physics A: Mathemathical and Theoretical, 40, 13063-13073. https://doi.org/10.1088/1751-8113/40/43/014

[2] Nininahazwe, A. (2018) Non-Hermitian Matrix Quasi-Exactly Solvable Hamiltonian. Open Journal of Microphysics, 8, 15-25.

https://doi.org/10.4236/ojm.2018.83003

[3] Nininahazwe, A. (2013) Matrix Quasi-Exactly Solvable Jacobi Elliptic Hamiltonian. Open Journal of Microphysics, 3, 53-59. https://doi.org/10.4236/ojm.2013.33010

[4] Brihaye, Y. and Nininahazwe, A. (2004) On PT-Symmetric Extensions of Calogero and Sutherland Models. International Journal of Modern Physics A, 19, 4391-4400. https://doi.org/10.1142/S0217751X04019858

[5] Turbiner, A.V. (1988) Quasi-Exactly Solvable Problems and sl(2) Algebra. Communications in Mathematical Physics, 118, 467-474.

https://doi.org/10.1007/BF01466727 
[6] Ushveridze, A.G. (1995) Quasi-Exactly Solvable Models in Quantum Mechanics. Institute of Physics Publishing, Bristol.

[7] Turbiner, A.V. (1989) Lamé Equation sl(2) Algebra and Isospectral Deformations. Journal of Physics A: Mathematical and General, 22, 1-144. https://doi.org/10.1088/0305-4470/22/1/001

[8] Shifman, M.A. and Turbiner, A.V. (1989) Quantal Problems with Partial Algebriazation of the Spectrum. Communications in Mathematical Physics, 126, 347-365. https://doi.org/10.1007/BF02125129

[9] Brihaye, Y. and Kosinski, P. (1997) Quasi-Exactly Solvable Matrix Models in sl(n). Physics Letters B, 424, 43-47. https://doi.org/10.1016/S0370-2693(98)00167-1

[10] Brihaye, Y. and Hartmann, B. (2001) Quasi-Exactly Solvable N $\times$ N-Matrix Schrödinger Operators. Modern Physics Letters A, 16, 1895-1906.

https://doi.org/10.1142/S0217732301005242 